\title{
The Research of Image Retrieval based on Multi Feature DS Evidence Theory Fusion
}

\author{
Che Chang ${ }^{1,2^{*}}$, Yu Xiaoyang ${ }^{1}$, Yu Guang ${ }^{1}$ \\ ${ }^{1}$ Measuring and Control Technology and Instrumentations \\ Harbin University of Science and Technology, \\ ${ }^{2}$ School of Engineering, Harbin University, Harbin, China \\ E-mail: chechang@163.com
}

\begin{abstract}
The traditional image retrieval method is based on single feature retrieval method like color, texture, shape or multi feature weighted fusion method. The retrieval rate of existing methods is not high. This paper presents fusion multi feature image retrieval. Two kinds of feature play a complementary effect in retrieval. Most color feature can not reflect the spatial information of image. Texture is a regional characteristics which contains important spatial information of image. Fusing the color and texture feature can play their respective advantages. At the same time, avoiding the one-sidedness of single feature retrieval. Improving the retrieval accuracy and flexibility well.
\end{abstract}

Keywords: color feature; texture feature; multi feature; fusion;

\section{Introduction}

The image type is in different flavors. Because the differences of information characteristics, the retrieval would be very considerably by each retrieval method. Sometimes, the color feature is obvious and the texture feature is not obvious in one image[1].If being retrieved by the color feature, the retrieval effect is better than which is retrieved by the texture feature. Similarly, when the texture feature is obvious and the color feature is not obvious in one image, we should use the texture feature to retrieve [2]. A lot of dissimilar images being retrieved by single feature, Their similarity measurement results are close yet. We would argue that were two similar images if we extract feature to do the similarity measurement by using the single color histogram. Because of the two similar images, we would the wrong search results. When the color and texture features are not obvious in one image, we need to find a new retrieval method.

In order to suit for all kinds of images, making full use of various information contained in images. It can meet the seeking of different users. Overcoming the single feature or two characteristics which are not very obvious. We use multi feature fusion to retrieve images, which can make the false detection probability in two dissimilar images reduce to the minimum. Even though it is high similarity in one feature, the other are not high similarity. It would be a very good improvement in the single trait defects. At the same time, According to the retrieval needs and the importance of each feature, the users can divide the fusion color and the weight of texture feature flexibly. So then it can achieve the best retrieval results.

\section{Dempster-Shafer Evidence Theory}

Evidence theory is a kind of inexact reasoning theory. There are many outstanding advantages which compared with the probability theory. While lacking of knowledge or

${ }^{*}$ Corresponding Author 
the theory is not clear, it is unable to give certainty judgment. It can not use the probability theory, but the evidence theory can be used in this kind of situation. It has broader defined requirements. Evidence theory is the probability in which the probability values is determined[3].It is a special case. Evidence theory is a widely used decision theory.

\subsection{Frame of Discernment}

Frame of discernment is a collection which can represent all elements in the hypothesis space. It is marked as $\theta$ usually. Each element in the collection is mutual exclusive. When there are $\mathrm{n}$ elements in the collection. $2^{\mathrm{n}}$ expresses the size of discernment.

For example, there is a discrimination frame $\theta:\{A, B, C\}$. Then all the hypothesis space set which it represents is $\{\Phi\},\{\mathrm{A}\},\{\mathrm{B}\},\{\mathrm{C}\},\{\mathrm{A}, \mathrm{B}\},\{\mathrm{A}, \mathrm{C}\},\{\mathrm{B}, \mathrm{C}\},\{\mathrm{A}, \mathrm{B}, \mathrm{C}\}$ [4].

\subsection{Basic Probability Assignment-BPA}

The basic probability assignment function is a certain value in range [0,1]. The size of this value shows the correlation between the evidence with assumed. It represents whether the choice of evidence body is right. It is marked as $m(A)$ usually. $A$ expresses some subsets in discernment frame. $m(A)$ need to satisfy the following formula.

$$
\left\{\begin{array}{l}
m(\Phi)=0 \\
\sum_{A \subseteq 2^{\theta}} m(A)=1
\end{array}\right.
$$

If the evidence and a putative $A$ is irrelevant, being marked as $1-m(A)$. It can be marked as $m_{A}(\theta)$. It means the accurate assignment evidence value can not be given to all subclass. It is the core idea of evidence theory [5]. There is a special discernment frame $\theta:\{A, B, C\}, \quad m(A)+m(B)+m(C)<1$. This definition and the probabilistic nature are totally different. The probability requirements is $\mathrm{P}(\mathrm{A})+\mathrm{P}(\mathrm{B})+\mathrm{P}(\mathrm{C})=1$. When the knowledge is inadequate or the theory is not clear, the judgement is uncertainty. The basic probability assignment function $m(\theta)$ can solve it. It can assign the probability values to any subset accurately.

\subsection{Belief Function}

When $2^{\theta} \in[0,1]$, the trust function Bel could be marked as:

$$
B e l=\sum_{B \subseteq A} m(B)
$$

Trust function Bel expresses the total trust degree to $A .2^{\theta}$ expresses the hypothesis space which is constituted by discernment frame. $\forall A \subseteq \theta$. According to the definition, $\operatorname{Bel}(\phi)=0, \operatorname{Bel}(\theta)=1$.

\subsection{Plausibility Function}

When $2^{\theta} \in[0,1]$, The likelihood function PL could be marked as:

$$
P L(A)=1-\operatorname{Bel}\left(A^{C}\right)=\sum_{B \cap A \neq \phi} m(B), \forall A \subset \theta, A^{C}=\theta-A
$$


Likelihood function expresses the trust degree of do not deny $A$.The relationship between the trust function and the likelihood function is $P L(A) \geq \operatorname{Bel}(A)$. The two can constitute the confidence interval of $A$ which is $[\operatorname{Bel}(A), P L(A)][6]$.

\section{The Experiment of Image Retrieval based on Multi Feature DS Evidence Theory Fusion}

\subsection{Dempster Rule of Evidential Combination}

According to the difference of getting evidence, there are avariety of BPA. In order to getting the accurate judgment of each event, It need to merge all the evidence body. We can use orthogonal method to merge. For example, it can use orthogonal sum to merge belief function Bell (BPA is $m_{1}$ ) with belief function Bel2(BPA is $m_{2}$ ). After combination, belief function $\mathrm{Bel}$ is:

$B e l=B e l 1 \oplus B e l 2$

In general, BPA of subset $\mathrm{A}$ is:

$m(A)=K^{-1} \sum_{A_{i} \cap B_{j}=A} m_{1}\left(A_{i}\right) m_{2}\left(B_{j}\right)$

In the formula, $K$ expresses the orthogonal constant. It expresses the contradiction degree of each evidence body in the same hypothesis. $K$ can refrain the probability values from being distributed to null set in unclear circumstances [7].

$K=1-\sum_{A_{i} \cap B_{j}=\phi} m_{1}\left(A_{i}\right) m_{2}\left(B_{j}\right)$

If there are multiple evidence body, like $B e l 1, B e l 2, \ldots, B e \ln$, the BPA of $A$ is $((B e l 1 \oplus \operatorname{Bel} 2) \oplus \ldots) \oplus B e \ln$. When merging evidence with DS rule, it must consider the following points:

(1) All the elements must be mutual independence in $\theta$, so it can meet the orthogonal operational properties. Sometimes the actual situation is not fit for purpose. It can use some transform methods to satisfy the independent conditions [8].

(2) Each belief function must be in the same discrimination frame.

(3) This rule is to use all the evidence body fully to judge. It can get probabilistic result which is more accurate and reliable.

(4) The orthogonal method is exchangeable. The merge order does not affect the results.

(5) When the value of $K^{-1}$ is bigger, It means the contradiction degree of data source between each evidence is greater.

(6) Disadvantage of the rule is that the increasing elements number in discrimination frame can make the computation more complicated. Hindering the development in the practical application. 


\subsection{Experimental Procedure}

For the image retrieval system based on the content, entering a sample image, it can export a result which is sorted by similarity degree. Each image make the color matching in image database. Also, each image in image database can export a result which is sorted by textural features [9]. After putting DS evidence theory to use, it can get a matching result which fuse color feature and texture feature.

In order to gain the superiority and accuracy of image retrieval which contrast with previous content-based methods, designing a experiment system of image retrieval based on multi feature DS fusion strategy. The development tool is Matlab7.0. The system extract the color feature and texture feature to the images which are in the image library. Constructing the image feature database. The color and texture feature vector of images in feature library would do the similarity matching. It can get the trust of color and texture feature about query images in image library. The vector trust of color and texture feature could be fused DS evidence theory consolidation method. It can get the general trust of each image. According the total trust degree fusing color feature and texture feature, the system would output the retrieval results.

The realization steps of image retrieval based on multi feature DS color and texture feature fusion is:

Step 1: Using the method of multi color feature combination. It can extract the color feature of query images. Using the method of multi scale Gabor wavelet transform. It can extract the texture feature of query images.

Step 2: Calculating the query images $Q$ by similarity measure. Detecting the color similarity distance $d(C 0, C i)$ between $Q$ with the $I$ image in image library. Besides, Detecting the texture similarity distance $d(T 0, T i)$ between $Q$ with the $I$ image in image library.

Step 3: If setting the trust of color feature and texture feature arbitrarily, it is bound to bring the retrieval error. In this paper, using the similarity distance of color feature $d(\mathrm{CO}, \mathrm{Ci})$ to ensure the color characteristics trust in each image. Using the similarity distance of texture feature $d(C 0, C i)$ to ensure the texture characteristics trust in each image.

Step 4: The color feature and texture feature is difference in physical meaning. Doing the feature vector normalization, in order to fuse two features. Two kinds of characteristics do the orthogonal calculation in the same sense.

Step 5: After getting the color and texture characteristics trust in each image, calculating the total trust degree of each image according to the formula $m(A)=K^{-1} \sum_{A_{i} \cap B_{j}=A} m_{1}\left(A_{i}\right) m_{2}\left(B_{j}\right)$.

Step 6: The retrieval results are being sorted by total trust degree. Exporting the similar image according to the specified requirements of users [10].

\section{Testing Results and Analysis}

\subsection{Image Retrieval Algorithm based on More Color Feature Combination}

Selecting Africa as target image to query. There are 100 images which are related to the target image in image database. The precision of using image retrieval based on more color feature combination is $80 \%$.The recall is $16 \%$, which is shown as Figure 1. 


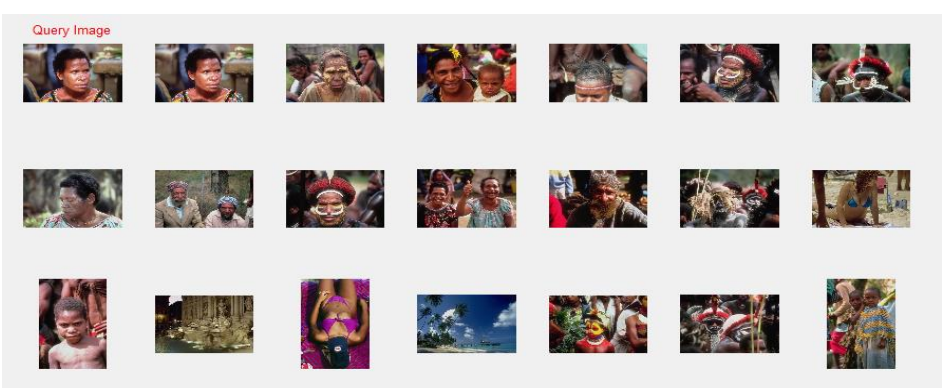

Figure 1. The Africa Image Retrieval Result of More Color Features

Selecting bus as target image to query. There are 100 images which are related to the target image in image database. The precision of using image retrieval based on more color feature combination is $70 \%$. The recall is $14 \%$, which is shown as Figure 2.

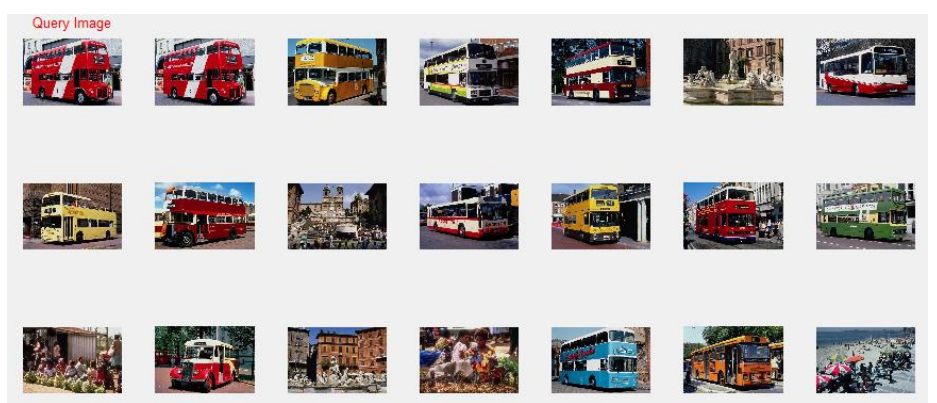

Figure 2. The Bus Image Retrieval Result of More Color Features

Selecting dinosaur as target image to query. There are 100 images which are related to the target image in image database. The precision of using image retrieval based on more color feature combination is $80 \%$. The recall is $16 \%$, which is shown as Figure 3.

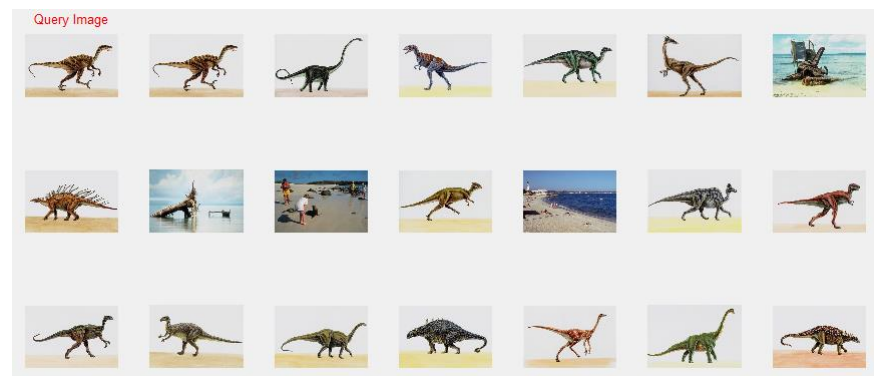

Figure 3. The Dinosaur Image Retrieval Result of More Color Features

Three different types of images experimental results is shown as Table 1:

Table 1. Adjacent Pixels Correlation Coefficient of Original and Cipher Images

\begin{tabular}{ccc}
\hline Type & Precision (\%) & Recall (\%) \\
\hline Africa & 80 & 16 \\
Bus & 70 & 14 \\
Dinosaur & 80 & 16 \\
Average & 76 & 15 \\
\hline
\end{tabular}




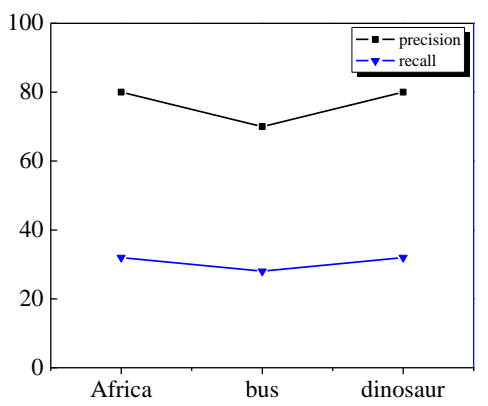

\section{Figure 4. The Image Retrieval Recall-Precision Criterion of More Color Features}

\subsection{Image Retrieval Algorithm based on Gabor Texture Feature Combination}

Selecting Africa as target image to query. There are 100 images which are related to the target image in image database. The precision of using image retrieval based on Gabor texture feature combination is $75 \%$.The recall is $15 \%$, which is shown as Figure 5.

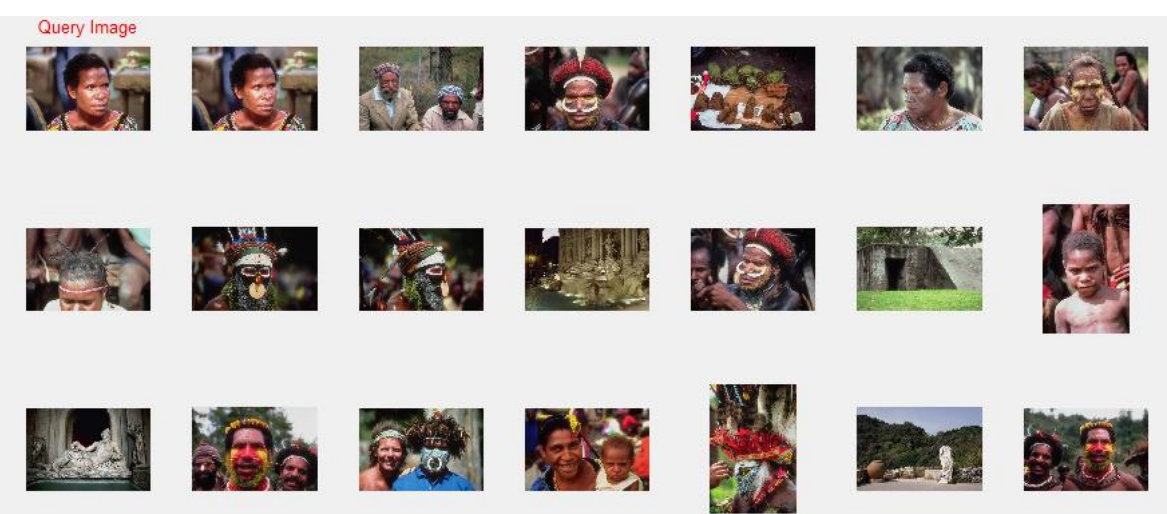

\section{Figure 5. The Image Retrieval Result of Africa based on Gabor Texture Features}

Selecting bus as target image to query. There are 100 images which are related to the target image in image database. The precision of using image retrieval based on Gabor texture feature combination is $80 \%$.The recall is $16 \%$, which is shown as Figure 6.

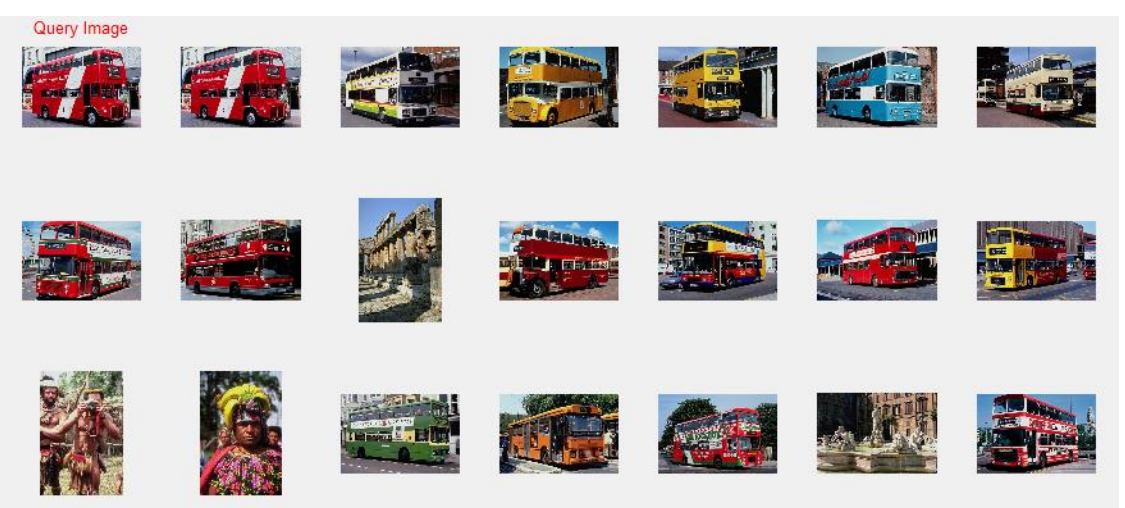

Figure 6. The Image Retrieval Result of Bus based on Gabor Texture Features 
Selecting dinosaur as target image to query. There are 100 images which are related to the target image in image database.The precision of using image retrieval based on Gabor texture feature combination is $95 \%$.The recall is $19 \%$, which is shown as Figure 7.

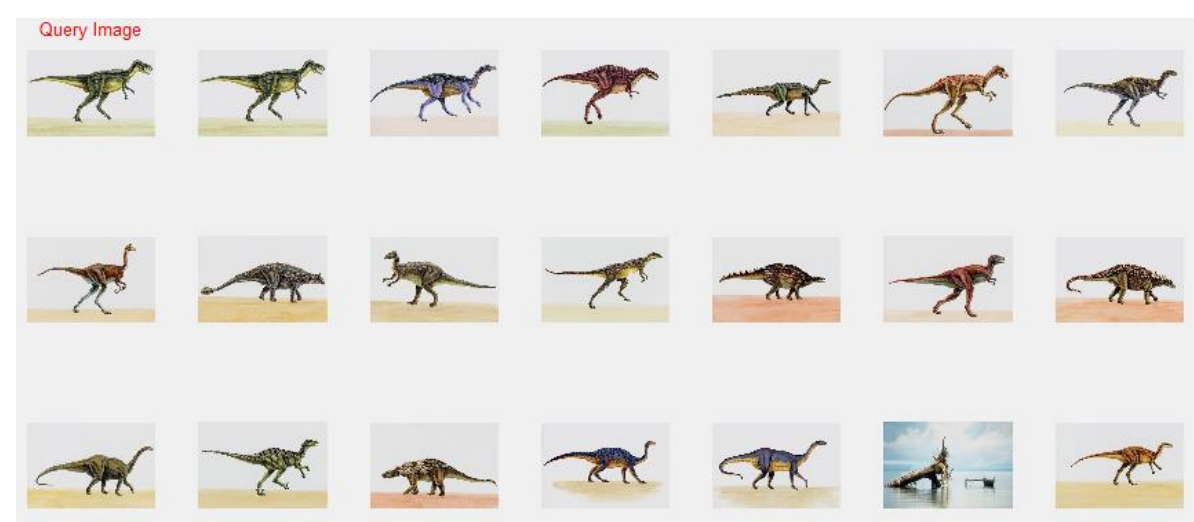

Figure 7. The Image Retrieval Result of Dinosaur based on Gabor Texture Features

Three different types of images experimental results is shown as Table 2:

Table 2. Adjacent Pixels Correlation Coefficient of Original and Cipher Images

\begin{tabular}{ccc}
\hline Type & Precision (\%) & Recall (\%) \\
\hline Africa & 75 & 15 \\
Bus & 80 & 16 \\
Dinosaur & 95 & 19 \\
Average & 83 & 17 \\
\hline
\end{tabular}

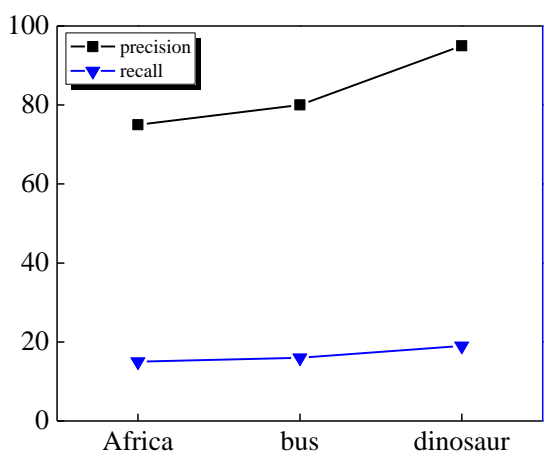

Figure 8. The Image Retrieval Recall-Precision Criterion based on Gabor Texture Features

\subsection{Image Retrieval Algorithm based on Integrated Multi Feature Combination}

We use Corel image library to do the fusion experiments similarly. The experimental results analysis method is the same with single color or texture feature. Using the same type of image retrieval. Exporting 20 images as the retrieval results in image library during the experiment. Calculating the image retrieval accuracy, which could do the 
image retrieval performance assessing criterion of fusion algorithm. Comparing with the experimental result of single color or texture feature. It can prove the accuracy and superiority of image retrieval based on multi feature DS evidence theory fusion. The retrieval results are shown as follows:

Selecting Africa as target image to query. There are 100 images which are related to the target image in image database. The precision of using image retrieval based on integrated multi feature combination is $90 \%$. The recall is $18 \%$, which is shown as Figure 9.

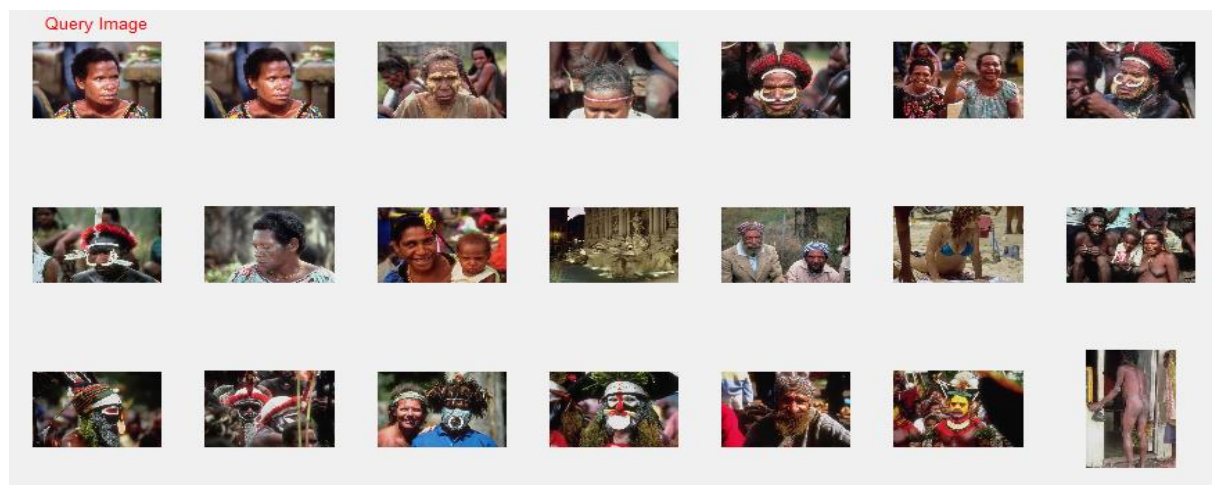

\section{Figure 9. The Image Retrieval Result of Africa based on Integrated Multi Feature}

Selecting bus as target image to query. There are 100 images which are related to the target image in image database. The precision of using image retrieval based on integrated multi feature combination is $90 \%$. The recall is $18 \%$, which is shown as Figure 10.

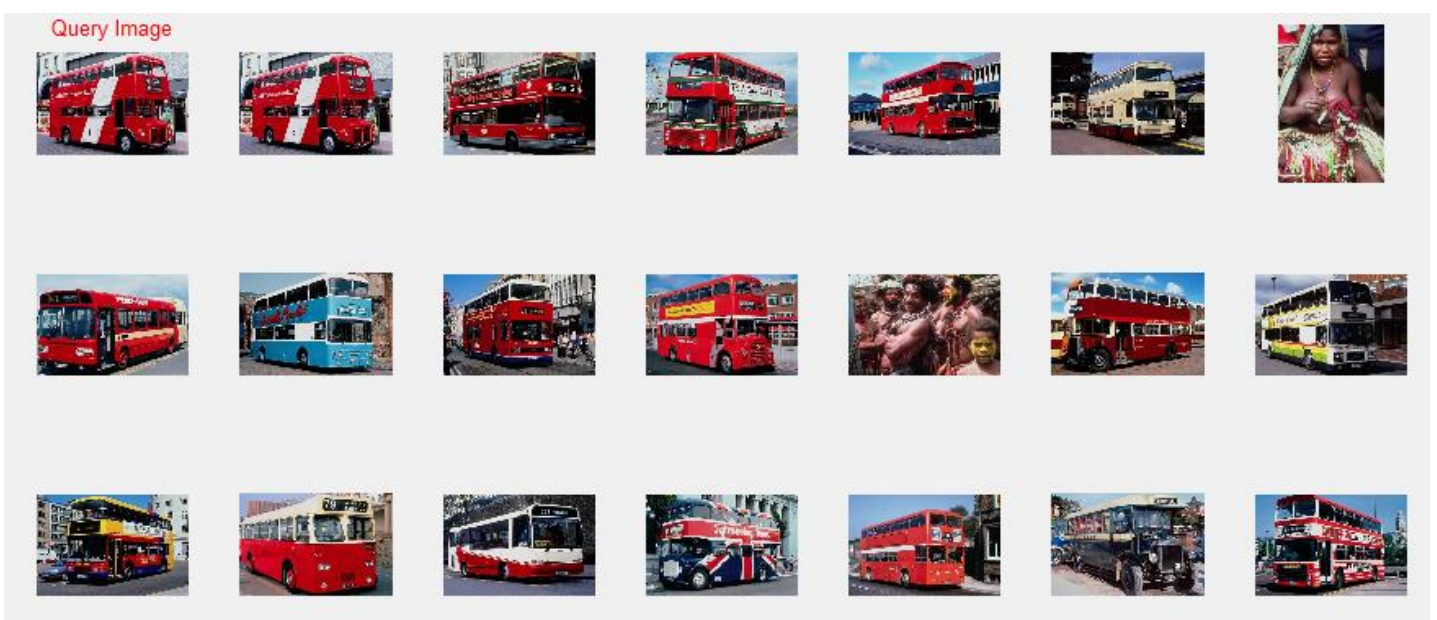

\section{Fig. 10 The image retrieval result of bus based on integrated multi feature}

Selecting dinosaur as target image to query. There are 100 images which are related to the target image in image database. The precision of using image retrieval based on integrated multi feature combination is $100 \%$. The recall is $20 \%$, which is shown as Figure 11 . 


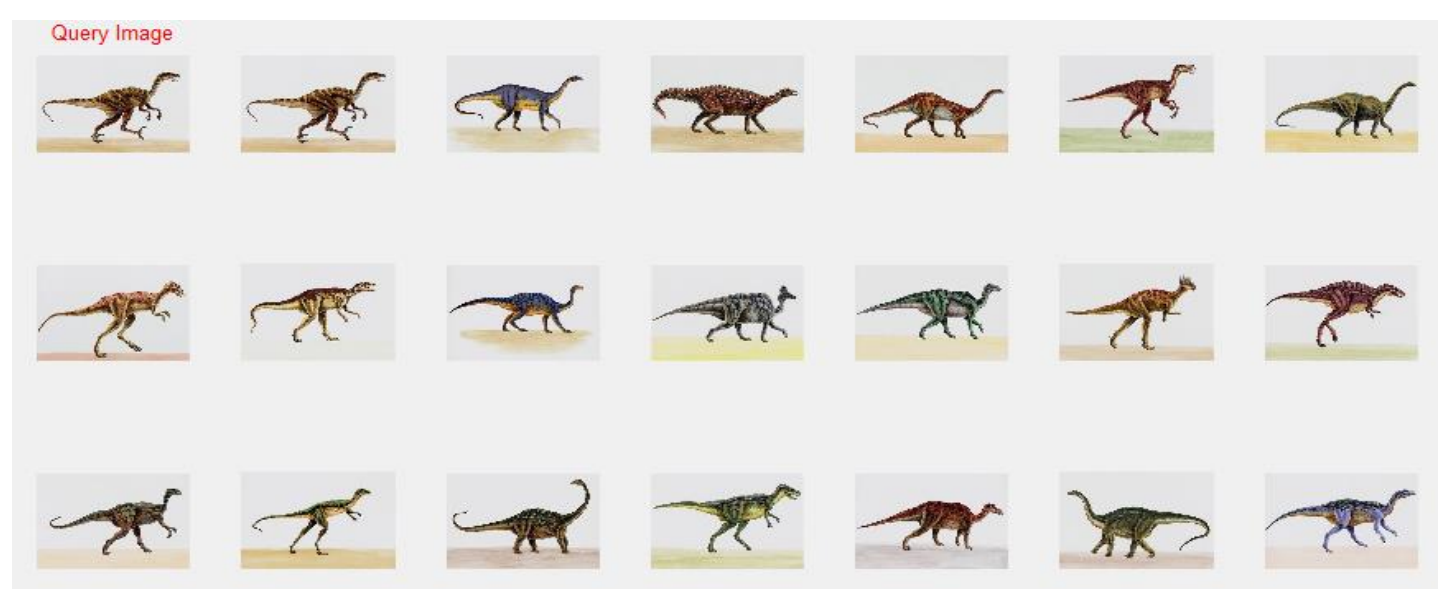

Figure 11. The Image Retrieval Result of Dinosaur based on Integrated Multi Feature

We evaluate the algorithm by using precision and recall for multi feature fusion experiment. According to the experimental results comparison, the accuracy rate of image retrieval algorithm based on multi feature fusion is higher than any single character retrieval algorithm. When the image color feature is obvious, the retrieval accuracy is low by texture feature retrieval. When the texture feature is obvious, the retrieval accuracy is low by color feature retrieval. The method could overcome these problems. Besides, we use DS theory merging rules to do the feature fusion. Overcoming the man-made error effectively, which the user determine the weight size according to the subjective. The image retrieval based on multi feature DS evidence theory fusion get a high precision in different feature types images. The multi feature fusion algorithm improves the accuracy of image retrieval greatly. It can overcome the shortcoming of single feature image retrieval effectively.

The retrieval algorithm experimental results based on color, texture and multi feature fusion in three different types of images are shown as follow:

\section{Table 3. Retrieval Performance Comparison based on Color, Texture and Integrated Multi Feature}

\begin{tabular}{ccccccc}
\hline & \multicolumn{2}{c}{ Color } & \multicolumn{2}{c}{ Texture } & \multicolumn{2}{c}{ Multi Feature } \\
Type & Precision(\%) & Recall $(\%)$ & Precision(\%) & Recall(\%) & Precision(\%) & Recall $(\%)$ \\
\hline Africa & 80 & 16 & 75 & 15 & 90 & 18 \\
Bus & 70 & 14 & 80 & 16 & 90 & 18 \\
Dinosaur & 80 & 16 & 95 & 19 & 100 & 20 \\
Average & 76 & 15 & 83 & 17 & 93 & 19 \\
\hline
\end{tabular}




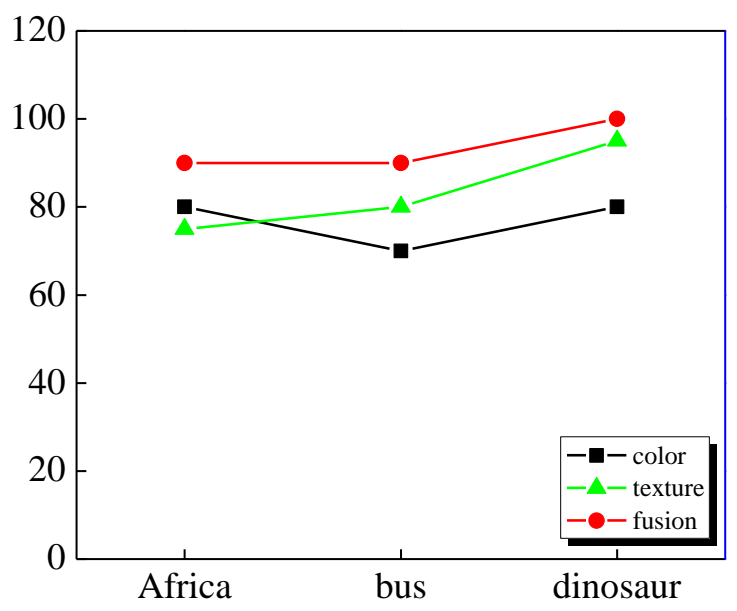

Figure 12. Precision Comparison based on Color, Texture and Integrated Multi Feature

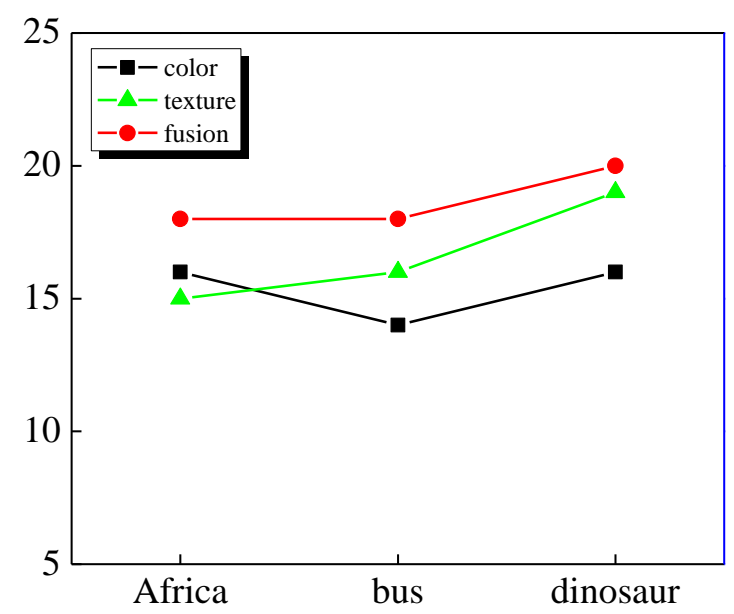

Figure 13. Recall Comparison based on Color, Texture and Integrated Multi Feature

The experimental results show that the retrieval results based on multi features DS evidence theory fusion is better than which based on single feature obviously.

\section{Conclusion}

This paper introduces the DS evidence theory. Mixing the color histogram, color moment, color auto correlogram and Gabor texture feature together. It builds the image retrieval algorithm and retrieval system based on multi DS fusion strategy. The retrieval experiment in Corel image database proves that the retrieval algorithm based on multi feature DS fusion strategy is better than retrieval algorithm based on single feature in retrieval performance. The average precision is $93 \%$. The average recall is $18.6 \%$. 


\section{References}

[1] W. Yang, "Optimization of Logistics Dispatching Route Theory Application and Research in Solid Matching Technic [D]", Journal of Harbin University of Science and Technology, (2011).

[2] Y. Liu, D. S. Zhang and G. Lu, "A survey of Content-Based Image Retrieval with High-Level Semantics[J]", Pattern Recognition, 2007, vol. 40, no. 1, (2007), pp. 262-282.

[3] L. chuen-Horng, C. Rong-Tai and c. yung-Kuan, "A smart Content-based Image Retrieval System Based on Color and Texture Feature[J]", Image and Vision Computer, 2009, vol. 27, no. 6, (2009), pp. 658-665.

[4] Christopher J. C. Burges, "A Tutorial on Support Vector Machines for Pattern Recognition [J]”, Data Mining and Knowledge Discovery, 1998, vol. 2, (1998), pp. 121-167.

[5] C. L. Tan, W. Huang, Z. Yu and Y. Xu, "Imaged document text retrieval without OCR[J]", IEEE Transactions on Pattern Analysis and Machine Intelligence, 2002, (2002).

[6] C. Wang, T. Chen, Y. Chan, R. H. wang and W. Huang, "Chinese document image retrieval system based on proportion of black pixel area in a character image[C]", Proc.6th ICACT, 2004, (2004).

[7] H. Muller, N. Michoux and D. Bandon, "A review of content-based image retrieval systems in medical applications-clinical benefits and future directions[J]", International Journal of Medical Informatics, 2004, vol. 73, (2004), pp. 1-23.

[8] G. Feng, Z. Nanning and S. Yonghong, 'Document images retrieval based on multiple features combination[C]”, IEEE ICDAR, 2007, (2007).

[9] M. S. Lew, N. Sebe, C. Djeraba and R. Jain, "Content-based multimedia information retrieval state of the art and challenges[J]", ACM Transactions on Multimedia Computing, Communications, and Applications, 2006, vol. 2, (2006), pp. 1-19.

[10] Y. Liu, D. S. Zhang and G. J. Lu, "A survey of content-based image retrieval with high-level semanticsl[J]”, Pattern Reorganization, 2007, vol. 40, (2007), pp. 262-282. 
International Journal of Signal Processing, Image Processing and Pattern Recognition Vol.9, No.1 (2016) 